\title{
Inversion of Stokes profiles with systematic effects
}

\author{
A. Asensio Ramos ${ }^{1,2}$, J. de la Cruz Rodríguez ${ }^{3}$, M. J. Martínez González ${ }^{1,2}$, and A. Pastor Yabar ${ }^{1,2}$ \\ 1 Instituto de Astrofísica de Canarias, 38205 La Laguna, Tenerife, Spain \\ e-mail: aasensio@iac.es \\ 2 Departamento de Astrofísica, Universidad de La Laguna, 38205 La Laguna, Tenerife, Spain \\ 3 Institute for Solar Physics, Dept. of Astronomy, Stockholm University, Albanova University Center, 10691 Stockholm, Sweden \\ e-mail: jaime@astro.su.se
}

Received 26 February 2016/ Accepted 16 April 2016

\begin{abstract}
Quantitative thermodynamical, dynamical and magnetic properties of the solar and stellar plasmas are obtained by interpreting their emergent non-polarized and polarized spectrum. This inference requires the selection of a set of spectral lines that are particularly sensitive to the physical conditions in the plasma and a suitable parametric model of the solar/stellar atmosphere. Nonlinear inversion codes are then used to fit the model to the observations. However, the presence of systematic effects, like nearby or blended spectral lines, telluric absorption, or incorrect correction of the continuum, among others, can strongly affect the results. We present an extension to current inversion codes that can deal with these effects in a transparent way. The resulting algorithm is very simple and can be applied to any existing inversion code with the addition of a few lines of code as an extra step in each iteration.
\end{abstract}

Key words. Sun: magnetic fields - Sun: atmosphere - line: profiles - methods: data analysis

\section{Introduction}

The decade of 1970s will be remembered as the one in which solar physicists were able to really start to infer the magnetic and thermodynamic properties of the solar plasma from observations. At that time, there was a nice coincidence. On the one hand, the theory of radiative transfer for polarized light was already in its maturity. On the other, computers started to be available for researchers in general and which were powerful enough to carry out complex calculations. It was at this time that the ideas of nonlinear inversion codes were set (Harvey et al. 1972; Auer et al. 1977; Skumanich \& Lites 1987).

Inversion algorithms are able to extract information about the magnetic and thermodynamical properties of the solar plasma from the analysis of spectropolarimetric observations. They function by proposing a specific model to explain the observations and then defining a merit function (usually the $\chi^{2}$ function, valid under the presence of uncorrelated Gaussian noise). The model parameters are iteratively modified for optimizing the merit function. The first inversion codes were relatively simple and based on strongly simplifying assumptions, like the MilneEddington (ME) approximation to analytically solve the radiative transfer equation (e.g., Harvey et al. 1972; Auer et al. 1977; Landi Degl'Innocenti \& Landolfi 2004). These inversion codes are still used today, like VFISV (Borrero et al. 2007, 2010), MILOS (Orozco Suárez et al. 2007) or MERLIN (Skumanich \& Lites 1987; Lites et al. 2007).

An enormous step forward was introduced by Ruiz Cobo \& del Toro Iniesta (1992), who developed Stokes inversion based on response functions (SIR), an inversion code that recovers the optical depth stratification of the physical quantities (temperature, magnetic field, velocity, etc.) from the interpretation of the Stokes profiles. This code is based on the idea of the response function (Landi Degl'Innocenti \& Landi Degl'Innocenti 1977), that allows the user to link the perturbation in the emergent Stokes parameters with perturbations in the physical parameters. One of the key ingredients that facilitated the development of these inversion codes was the possibility to find an analytical expression for the response functions in local thermodynamic equilibrium (LTE; Sanchez Almeida 1992; Ruiz Cobo \& del Toro Iniesta 1994). Based on the essence of SIR, several nonlinear inversion codes are now available, some of them even dealing with the much more difficult case of the inversion of spectral lines in non-LTE (NICOLE; Socas-Navarro et al. 2000, 2015). All these 1D inversion codes are based on the concept of so-called nodes, that need to be defined a priori. These nodes mark positions along the optical depth axis where the value of the physical parameters will be modified to fit the Stokes profiles. The full stratification of the atmosphere between the nodes, which is needed to accurately integrate the radiative transfer equation and to derive the gas pressure scale, is interpolated using a piecewise polynomial. The complexity of the solution then critically depends on the number of nodes that are employed to describe each of the physical parameters.

After more than a decade without any fundamental improvement (except perhaps the introduction of Bayesian inference into the field; Asensio Ramos et al. 2007, 2012; Asensio Ramos 2009), we are now going through another interesting era, again driven by the improvements in computational power. On the one hand, van Noort (2012) has developed a spatially coupled twodimensional inversion code in which the effect of the telescope point spread function (PSF) is taken into account. The PSF couples nearby pixels so that deconvolution and inversion is done at the same time. On the other hand, following a similar motivation, Ruiz Cobo \& Asensio Ramos (2013) have used a regularized deconvolution of the Stokes profiles based on the principal component analysis (PCA) and have used SIR to invert the deconvolved Stokes parameters. 

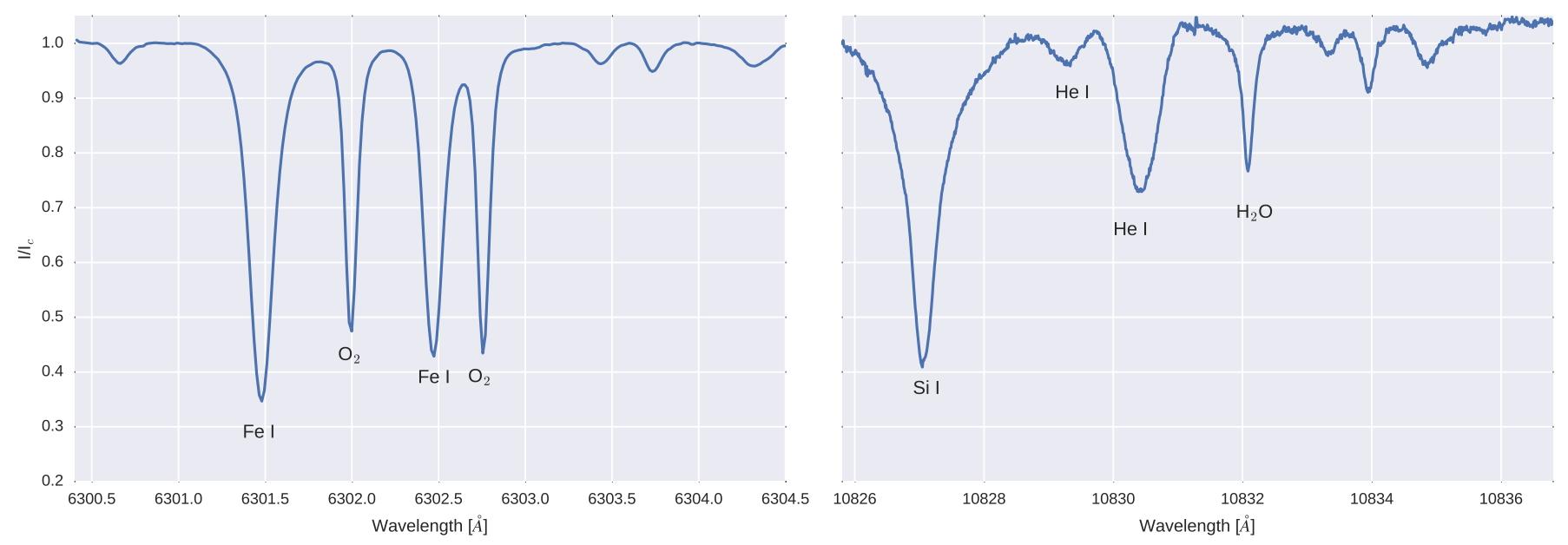

Fig. 1. Examples of situations in which systematic effects are important when extracting information from spectral lines.

Arguably, the last step in the evolution of inversion codes has been the introduction of regularization ideas based on the concept of sparsity or compressibility ${ }^{1}$ (e.g., Starck et al. 2010). Asensio Ramos \& de la Cruz Rodríguez (2015) have presented an inversion code for the inversion of Stokes profiles that uses $\ell_{0}$ or $\ell_{1}$ regularization on a transformed space ${ }^{2}$. The two-dimensional maps of parameters are linearly transformed (Fourier, wavelet, or any other appropriate transformation can be used) and assumed to be sparse in the transformed domain. This introduces two important constraints. First, the sparsity assumption reduces the number of unknowns in the problem, avoiding overfitting. Second, the global character of the transformations that are routinely used, spatial correlation of the result is automatically taken into account. Consequently, the Stokes parameters observed at every pixel potentially introduces constraints onto every other pixel of the observed map. This is the first time that the inherent spatial correlation of the physical parameters has been taken into account in inversion codes. This results in much more stable inversion codes that do not produce spurious pixel-by-pixel variations of the maps of physical quantities. Additionally, the complexity of the solution is automatically adapted, producing more structure where it is needed.

In the conclusions of Asensio Ramos \& de la Cruz Rodríguez (2015), we point out that the sparsity regularization can be applied to invert Stokes profiles with systematic effects. This is precisely what we present in this paper. A customary way of dealing with systematic effects in current inversions is to downweight the influence on the merit function of the parts of the spectrum that are affected by these effects. Although it works in practice, it depends on a set of parameters (e.g., region and factor of the downweighting), which make it quite subjective. Instead, we propose several basis sets (orthogonal and non-orthogonal) to absorb the systematic effects (for instance, telluric lines) in the Stokes profiles and use a proximal projection algorithm (e.g., Parikh \& Boyd 2014) to make the solution automatically adapt to the necessary complexity. We show that the modifications needed in existing inversion codes are minimal so that this approach can be introduced without much effort.

\footnotetext{
1 Given an $n$-dimensional vector $\boldsymbol{x}$, sparsity means that the majority of the elements of the vector are strictly zero, while compressibility means that the elements of this vector, when ordered by absolute value, fulfill $\left|x_{i}\right| \leq C i^{-1 / r}$.

2 The $\ell_{p}$-norm is given by: $\|\boldsymbol{x}\|_{p}=\left(\sum_{i}\left|x_{i}\right|^{p}\right)^{1 / p}$, with $p \geq 0$.
}

\section{Sparsity regularization}

Our objective is to fit the observed Stokes parameters, which are discretized at $N$ finite wavelength points $\lambda_{j}$. For simplicity, we stack the four Stokes parameters in a long vector of length $4 N$ so that $\boldsymbol{O}=\left[I\left(\lambda_{1}\right), \ldots, I\left(\lambda_{N}\right), Q\left(\lambda_{1}\right), \ldots\right.$, $\left.U\left(\lambda_{1}\right), \ldots, V\left(\lambda_{1}\right), \ldots, V\left(\lambda_{N}\right)\right]^{\dagger}$ (with $\dagger$ the transpose). The fit is carried out using a model atmosphere, with the aim of extracting useful thermal, dynamic, and magnetic information from them. Additionally, we make the assumption that these Stokes parameters are perturbed by some uncontrollable systematic effects. These systematic effects can be, for instance, telluric lines produced by absorption in the Earth atmosphere, variations along the spectral direction produced by an incorrect illumination of the camera or a low-quality flatfielding, etc. Examples of these situations are found in Fig. 1. The left panel shows the wellknown region around $6302 \AA$ that contains two Fe I lines, which are blended with two telluric lines. This is of special importance for the Fe I line at $6302.5 \AA$. Extracting physical information from the line requires then to avoid the far wings, which can lead to problems in the deepest parts of the atmosphere. The right panel shows the region around $10830 \AA$, containing an $\mathrm{He}$ I multiplet used for chromospheric diagnostics, which is blended with a photospheric Si I line and a telluric line. The interpretation of the He I multiplet, given that it forms on the extended wings of the $\mathrm{Si}$ I line, usually requires a previous analysis of the photospheric line. Additionally, the strong chromospheric dynamics induces that the He I multiplet sometimes blends with the telluric absorption.

In very general terms, we can explain the observations with the following generative model:

$\boldsymbol{O}=\boldsymbol{S}_{\mathrm{atm}}(\boldsymbol{q})+\mathrm{D} \alpha+\boldsymbol{\epsilon}$

where $\alpha$ is a vector of dimension $d$ that describes the systematic effects via a dictionary $\mathbf{D} \in \mathbb{R}^{4 N \times d}$, also known as synthesis operator. We recall that a dictionary is just a collection of (possibly non-orthogonal) functions that are used to describe the systematic effects. The contribution $\boldsymbol{S}_{\text {atm }}(\boldsymbol{q})$ contains the Stokes parameters that emerge from a model atmosphere, which depends on a vector of model parameters $\boldsymbol{q}$, ordered like in $\boldsymbol{O}$. The noise components $\epsilon$ are considered to be Gaussian random variables with zero mean and diagonal covariance matrix. We note that when the size of the dictionary equals the number of observed spectral 
points $(d=4 N)$ and $\mathbf{D}=\mathbf{1}$ (with $\mathbf{1}$ the identity matrix), the model for the systematic effects described in Eq. (1) turns out to be very flexible because $\alpha$ refers to the particular value of the systematic effects at each sampled wavelength point.

From this generative model, the merit function that has to be optimized is the $\chi^{2}$ with diagonal covariance, in our case given by

$\chi_{\mathbf{D}}^{2}(\boldsymbol{q}, \boldsymbol{\alpha})=\frac{1}{4 N} \sum_{j=1}^{4 N} w_{j} \frac{\left[S_{\mathrm{atm}, j}(\boldsymbol{q})+(\mathbf{D} \boldsymbol{\alpha})_{j}-O_{j}\right]^{2}}{\sigma_{j}^{2}}$,

where we make explicit the dependence of the merit function on the election of the dictionary. The previous merit function considers a potentially different noise variance $\sigma_{j}^{2}$ for every Stokes parameter and wavelength position, which is surely the case for very strong lines in which the number of photons in the core is much more absorbed than the wings. Additionally, it is customary to introduce weights $w_{j}$ for each Stokes parameter to increase the sensitivity to some parameters when carrying out the inver$\operatorname{sion}^{3}$. These weights are precisely the ones that are currently used to deal with systematic effects, by decreasing their values for specific wavelength points.

The inferred physical parameters are typically found by solving the following maximum-likelihood problem:

$\underset{\boldsymbol{q}, \boldsymbol{\alpha}}{\arg \min } \chi_{\mathbf{D}}^{2}(\boldsymbol{q}, \boldsymbol{\alpha})$,

where the operator $\arg$ min returns the value of $\boldsymbol{q}$ and $\boldsymbol{\alpha}$ that minimizes the $\chi_{\mathbf{D}}^{2}(\boldsymbol{q}, \boldsymbol{\alpha})$ function. We note that Eq. (3) is, in general, non-convex ${ }^{4}$ for the inversion of Stokes parameters. Therefore, we only aspire to reach one of the local minima and later check for the physical relevance of the solution. Problem 3 is usually solved by a direct application of the Levenberg-Marquardt (LM) algorithm (Levenberg 1944; Marquardt 1963), which is especially suited to the optimization of these types of $\ell_{2}$-norms. Although Problem 3 is stated without any constraint, it is clear from the introduction that it is customary to regularize the solution by imposing some regularity of the solution through the use of nodes. Consequently, the vector of parameters $\boldsymbol{q}$ contains the value of the physical parameters (temperature, magnetic field, velocity etc.) at a small number of depths in optical depth in the atmosphere. In between these points, the physical properties are interpolated from the values at these nodes.

Without any additional constraint for $\alpha$, it is clear that we will encounter cross-talk between $\boldsymbol{\alpha}$ and $\boldsymbol{q}$. The fundamental reason is that our regressor for the systematic effects is so flexible that it can potentially fit the observations perfectly, including the specific noise realization. As noted above, if $d=4 N$ and $\mathbf{D}=\mathbf{1}$, a solution to Eq. (3) is $\boldsymbol{\alpha}=\boldsymbol{O}$ and $\boldsymbol{S}_{\mathrm{atm}}(\boldsymbol{q})=0$ for every observed wavelength. This trivial solution is of no interest because it does not extract any relevant physical information from the observations. To overcome this possibility, we follow recent ideas (Candès et al. 2006; Starck et al. 2010; Asensio Ramos \& de la Cruz Rodríguez 2015) and regularize the problem by imposing a sparsity constraint on $\alpha$ or, in general, on any transformation of $\boldsymbol{\alpha}$. Two fundamental approaches to include

\footnotetext{
3 Note that the role of the weights is to modify the value of the noise variance. If the noise variance is artificially increased for one Stokes parameter, its constraining power decreases.

4 When the function to minimize is convex in the variables, a local minimum is also the global minimum. This cannot be guaranteed in the optimization of non-convex functions, which is typically the case in the inversion of Stokes parameters.
}

this sparsity penalty have been proposed (Starck et al. 2010). Each one has advantages and disadvantages, as we will show in Sect. 4.

\subsection{The analysis penalty approach}

This approach is based on having as many degrees of freedom as observed data points $(d=4 N)$ and $\mathbf{D}=\mathbf{1}$ and solving the following problem:

$\underset{\boldsymbol{q}, \boldsymbol{\alpha}}{\arg \min } \chi_{\mathbf{1}}^{2}(\boldsymbol{q}, \boldsymbol{\alpha})$, subject to $\|\mathbf{W} \boldsymbol{\alpha}\|_{p} \leq s$,

where $\mathbf{W}$ is the matrix associated with any linear transformation of interest, either orthogonal or not, while $s$ is a predefined threshold. We also reiterate that $\|x\|_{p}=\left(\sum_{i}\left|x_{i}\right|^{p}\right)^{1 / p}$ is the $\ell_{q}$-norm. For instance, the $\ell_{0}$ norm of a vector is just the number of non-zero elements, while the $\ell_{1}$ norm is the sum of the absolute value of its elements. In other words, solving Eq. (4) requires us to seek the pair $(\boldsymbol{q}, \boldsymbol{\alpha})$ that better fits our observations, which imposes that the projection of the systematic effects on the transformed domain defined by $\mathbf{W}$ is sparse. The so-called analysis penalty comes from the fact that $\mathbf{W}$ is the analysis operator, which carries the vector $\boldsymbol{\alpha}$ to the transformed sparsity-inducing domain (Elad et al. 2007; Starck et al. 2010). It is especially suited to deal with cases in which $\mathbf{W}$ is non-orthogonal and/or overcomplete.

The solution to the previous problem when $p=0$ (or equivalently when $p=1$ under some conditions; Candès et al. 2006; Donoho 2006) is known to coincide with the exact solution when it exists. Sparsity is, therefore, a very convenient regularization. The only degree of freedom is to find the appropriate transformation $\mathbf{W}$. The same problem can be written equivalently with the addition of a regularization parameter $(\lambda)$ :

$$
\underset{\boldsymbol{q}, \boldsymbol{\alpha}}{\arg \min }\left[\frac{1}{4 N} \sum_{j=1}^{4 N} w_{j} \frac{\left[S_{\mathrm{atm}, j}(\boldsymbol{q})+\alpha_{j}-O_{j}\right]^{2}}{\sigma_{j}^{2}}+\lambda\|\mathbf{W} \boldsymbol{\alpha}\|_{p}\right] .
$$

\subsection{The synthesis penalty approach}

This approach is based on having $\mathbf{D}=\mathbf{W}^{\mathrm{T}}$ and imposing a sparsity constraint on the $\alpha$ vector itself. Consequently, we have to solve the problem

$\underset{\boldsymbol{q}, \boldsymbol{\alpha}}{\arg \min } \chi_{\mathbf{W}^{\mathrm{T}}}^{2}(\boldsymbol{q}, \boldsymbol{\alpha})$, subject to $\|\boldsymbol{\alpha}\|_{p} \leq s$,

which, in Lagrangian form, becomes

$$
\underset{\boldsymbol{q}, \boldsymbol{\alpha}}{\arg \min }\left[\frac{1}{4 N} \sum_{j=1}^{4 N} w_{j} \frac{\left[S_{\mathrm{atm}, j}(\boldsymbol{q})+\left(\mathbf{W}^{\mathrm{T}} \boldsymbol{\alpha}\right)_{j}-O_{j}\right]^{2}}{\sigma_{j}^{2}}+\lambda\|\boldsymbol{\alpha}\|_{p}\right]
$$

The name synthesis penalty comes from the fact that $\mathbf{W}^{\mathrm{T}}$ is the synthesis operator, which generates the systematic effects from the vector $\alpha$ living on the sparsity-inducing transformed domain.

Elad et al. (2007) note that both approaches are equivalent when $\mathbf{W}$ is an orthogonal transform (like Fourier, wavelet, etc.), because it fulfills $\mathbf{W}^{\mathrm{T}} \mathbf{W}=\mathbf{1}$. However, they solve completely different problems when $\mathbf{W}$ is non-orthogonal. 


\subsection{Transformations}

In this paper, we consider three options for the regularization term. The first one uses as regularization an orthogonal wavelet transform. The fact that $\mathbf{W}$ is orthogonal will slightly simplify the algorithms. The second one is a non-orthogonal overcomplete isotropic undecimated wavelet transform using the $\mathrm{B}_{3}$-spline (Starck et al. 2010). Finally, we will use a hand-made non-orthogonal transform made of Voigt functions centered at specific locations in the spectrum, which will be used to absorb the systematic effects. We defer the detailed description of each one until Sect. 3.3.

\section{The proposed optimization}

Given the special structure of both problems defined in Eqs. (5) and (7), in which the regularization only occurs for the $\boldsymbol{\alpha}$ variables, we propose to use an alternating optimization method. If $\alpha$ is fixed, Eq. (5) becomes the traditional least-squares problem for $\boldsymbol{q}$, which is solved efficiently with Newton-type methods like the Levenberg-Marquardt algorithm. This method uses secondorder information given by $\mathbf{H}_{q}$, the Hessian of the merit function with respect to the $\boldsymbol{q}$ variables:

$\boldsymbol{q}_{i+1}=\boldsymbol{q}_{i}-\hat{\mathbf{H}}_{q}^{-1} \nabla_{q} \chi_{\mathbf{1}}^{2}\left(\boldsymbol{q}_{i}, \boldsymbol{\alpha}_{i}\right), \quad$ with $\boldsymbol{\alpha}_{i}$ fixed.

Conforming to the prescriptions of the Levenberg-Marquardt algorithm, we use a modified Hessian matrix by enhancing its diagonal by a factor $\beta$, so that $\hat{\mathbf{H}}_{q}=\mathbf{H}_{q}+\beta \operatorname{diag}\left(\mathbf{H}_{q}\right)$. This hyperparameter is modified during the iteration to shift between the gradient descent method (large $\beta$ ) and Newton-type method $($ small $\beta)$. We note that inverting the Hessian matrix with a truncated singular value decomposition (Ruiz Cobo \& del Toro Iniesta 1992) introduces an extra regularization on the physical parameters at the nodes that is often needed.

\subsection{The analysis penalty}

On the other hand, when $\boldsymbol{q}$ is fixed, Eq. (5) becomes a standard sparsity-constrained linear problem for $\alpha$. This problem can be solved efficiently using proximal algorithms (Parikh \& Boyd 2014; Asensio Ramos \& de la Cruz Rodríguez 2015), which are especially suited to solving problems of the type:

$\underset{\boldsymbol{\alpha}}{\arg \min } f(\boldsymbol{\alpha}):=g(\boldsymbol{\alpha})+h(\boldsymbol{\alpha})$,

where $g(\boldsymbol{\alpha})=\chi_{\mathbf{1}}^{2}(\boldsymbol{q}, \boldsymbol{\alpha})$, is a smooth function and $h(\boldsymbol{\alpha})=\lambda\|\mathbf{W} \boldsymbol{\alpha}\|_{p}$ is a convex but not necessarily smooth function (we note that the derivative of the $\|\mathbf{W} \boldsymbol{\alpha}\|_{p}$ term is not continuous). We propose the following first-order iterative scheme to solve the problem:

$\boldsymbol{\alpha}_{i+1}=\operatorname{prox}_{p, \lambda, \mathbf{W}}\left[\boldsymbol{\alpha}_{i}-\tau \nabla_{\alpha} \chi_{\mathbf{1}}^{2}\left(\boldsymbol{q}_{i+1}, \boldsymbol{\alpha}_{i}\right)\right] \quad$ with $\boldsymbol{q}_{i+1}$ fixed,

where the operator $\operatorname{prox}_{p, \lambda, \mathbf{W}}$ is the proximal projection operator (Parikh \& Boyd 2014) associated with the constraint $\|\mathbf{W} \boldsymbol{\alpha}\|_{p}$, which we show how to efficiently compute in Sect. 4 . The election of the step size $\tau$ is important for the convergence of the algorithm. This is known to converge, provided the step size fulfills $\tau<2 /\left\|\mathbf{H}_{\alpha}\right\|^{2}$, where $\left\|\mathbf{H}_{\alpha}\right\|$ is the spectral norm of the Hessian of the merit function with respect to $\alpha$ (given by the square root of the maximum eigenvalue of $\mathbf{H}_{\alpha}^{\mathrm{T}} \mathbf{H}_{\alpha}$ ). We note that faster algorithms like FISTA (Beck \& Teboulle 2009; Asensio Ramos \& de la Cruz Rodríguez 2015) can also be used.
Given the simple dependence of $\chi_{\boldsymbol{q}, \boldsymbol{\alpha}}^{2}$ on $\boldsymbol{\alpha}$, it can be proven (e.g., Starck et al. 2010) that Eq. (10) can be simplified to finally obtain:

$\boldsymbol{\alpha}_{i+1}=\operatorname{prox}_{p, \lambda, \mathbf{W}}\left[\boldsymbol{S}_{\mathrm{atm}}\left(\boldsymbol{q}_{i+1}\right)-\boldsymbol{O}\right]$ with $\boldsymbol{q}_{i+1}$ fixed.

In other words, the estimation of the systematic effects for a new iteration is very simple and reduces to computing the proximal projection of the residual between the observed Stokes profiles and the current modeled ones. We think that this approach provides a very transparent and intuitive understanding of what the algorithm is doing. We note that the best results have been found applying Eq. (11) every $\sim 3$ iterations of the LM algorithm.

\subsection{The synthesis penalty}

When $\boldsymbol{q}$ is fixed, Eq. (7) again becomes a sparsity-constrained problem for $\boldsymbol{\alpha}$. In this case, the sparsity penalty $h(\boldsymbol{\alpha})=\|\boldsymbol{\alpha}\|_{p}$ is much simpler, but the function $g(\boldsymbol{\alpha})=\chi_{\mathbf{W}^{\mathrm{T}}}^{2}(\boldsymbol{q}, \boldsymbol{\alpha})$ is more complex because of the presence of the synthesis operator $\mathbf{W}^{\mathrm{T}}$. We propose solving the problem using the following first-order iterative scheme:

$\boldsymbol{\alpha}_{i+1}=\operatorname{prox}_{p, \lambda, \tau}\left[\boldsymbol{\alpha}_{i}-\tau \nabla_{\alpha} \chi_{\mathbf{W}^{\mathrm{T}}}^{2}\left(\boldsymbol{q}_{i+1}, \boldsymbol{\alpha}_{i}\right)\right] \quad$ with $\boldsymbol{q}_{i+1}$ fixed,

where $\tau<2 /\left\|\mathbf{H}_{\alpha}\right\|^{2}$, which can be computed from the spectral norm of the transformation matrix $\mathbf{W}$, and the proximal operator $\operatorname{prox}_{p, \lambda, \tau}$ is described in the following. We point out that a more complex second-order iterative scheme is discussed in the Appendix.

\subsection{Computing the proximal operators}

The previous iterative schemes rely on the existence of algorithms for the computation of the proximal projection operators $\operatorname{prox}_{p, \lambda, \tau}(\boldsymbol{\alpha})$ and $\operatorname{prox}_{p, \lambda, \mathbf{W}}(\boldsymbol{\alpha})$.

\subsubsection{Computing $\operatorname{prox}_{p, \lambda, \tau}(\alpha)$}

This operator is very simple to compute for typical choices of $p$ (Parikh \& Boyd 2014). Useful cases of the regularization term are the $\ell_{0}$-norm $(p=0)$ or the $\ell_{1}$-norm $(p=1)$. In the case of the $\ell_{0}$-norm, the proximal operator reduces to the hard-thresholding operator, which is trivially given by

$\operatorname{prox}_{p=0, \lambda, \tau}(\alpha)= \begin{cases}\boldsymbol{\alpha} & |\boldsymbol{\alpha}|>\tau \lambda \\ \mathbf{0} & \text { otherwise. }\end{cases}$

where $\tau$ is the step-size defined in Sect. 3.1 and $\lambda$ is the regularization parameter that we introduced in Eq. (5). For the $\ell_{1}$-norm, it reduces to the soft-thresholding operator, which is given by

$\operatorname{prox}_{p=1 \lambda, \tau}(\boldsymbol{\alpha})=\operatorname{sign}(\boldsymbol{\alpha})(|\boldsymbol{\alpha}|-\tau \lambda)_{+}$,

where $(\cdot)_{+}$denotes the positive part. Other proximal operators with analytical expressions can be found in Parikh \& Boyd (2014).

\subsubsection{Computing $\operatorname{prox}_{p, \lambda, w}(\alpha)$}

The solution of Eq. (11) for any $\mathbf{W}$, either orthogonal or not, is slightly more complicated and requires some elements of proximal calculus (Parikh \& Boyd 2014). A general algorithm for the solution of the proximal projection of Eq. (11) has been developed by Fadili \& Starck (2009), which we reproduce in Alg. 1 


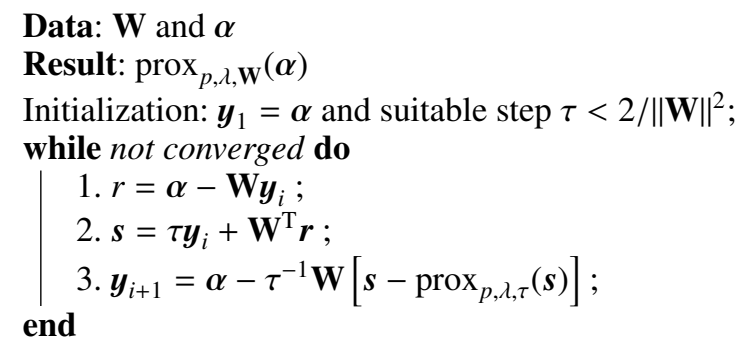

return $y_{i}$

Algorithm 1: Algorithm for proximal projection, extracted from Fadili \& Starck (2009).

for completeness. We note that the algorithm is just a simple iterative scheme that has been proven to converge with the solution, provided the step size $\tau<2 /\|\mathbf{W}\|^{2}$.

It is interesting to note that when $\mathbf{W}$ is an orthogonal transform, Alg. 1 hugely simplifies and the solution to Eq. (11) can be obtained with (Starck et al. 2010):

$\boldsymbol{\alpha}=\mathbf{W}^{\mathrm{T}}\left[\operatorname{prox}_{p, \lambda, \tau}(\mathbf{W} \boldsymbol{\alpha})\right]$.

In other words, the vector $\boldsymbol{\alpha}$ is first transformed, then it is thresholded using the appropriate proximal operator, and finally transformed back to the original domain. If the transformation is unitary, so that $\mathbf{W}^{\mathrm{T}} \mathbf{W}=\mathbf{1}$, the result of the application of Eq. (15) leaves the value of $\alpha$ unchanged for $\lambda=0$.

Another interesting use of Eq. (15) is that it is equivalent to the first iteration of Alg. 1. We carry out experiments in the following sections to verify if using this simple approximation in the general case of non-orthogonal $\mathbf{W}$ gives good results.

\subsection{Selection of $\lambda$}

As we show in the next section, $\lambda$ has a strong impact on the sparsity of the final solution. When $\lambda$ is too small, overfitting clearly appears. On the contrary, if $\lambda$ is large, the fit is typically of bad quality. Therefore, the selection of $\lambda$ requires some finetuning, but it is possible to have an order of magnitude estimation using very simple arguments. As noted in Eqs. (13) and (14), the thresholding happens for values of the $\alpha$ parameters that are larger than $\tau \lambda$. Therefore, if we want to avoid values of $\boldsymbol{\alpha}$ that are smaller than $\eta$, then $\lambda \sim \eta\|\mathbf{W}\|^{2}$. For the orthogonal unitary transforms, we find that the spectral norm is $\|\mathbf{W}\|=1$.

\subsection{Summary}

The full step-by-step algorithms are described in Algs. 2 and 3 , together with Alg. 1 for the application of the proximal operator described in step 4 of Alg. 2. We want to clarify to any potential user of this method that the only real difference between current inversion codes for the Stokes parameters and our approach is in point 4 of $\mathrm{Alg}$. 2, together with the necessity to include the systematic effects in the calculation of the gradient and Hessian with respect to the $\boldsymbol{q}$ variables. This step is very easy to carry out and can be implemented in any existing inversion code with only a few lines of programming.

\section{Examples}

In this section we demonstrate the capabilities of Algs. 2 and 3 in the inversion of Stokes profiles using several transformation matrices $\mathbf{W}$ and two datasets. For simplicity and for the purpose
Data: Stokes profiles, model atmosphere, transform $\mathbf{W}$ and $k$.

Result: Regularized solution

Initialization: $\boldsymbol{q}_{0}$ and $\boldsymbol{\alpha}_{0}=0$, first estimation of solution; while not converged do

1. Compute gradient $\nabla_{q} \chi_{\mathbf{1}}^{2}\left(\boldsymbol{q}_{i}, \boldsymbol{\alpha}_{i}\right)$ and Hessian matrices $\mathbf{H}_{q}$;

2. Modify Hessians: $\hat{\mathbf{H}}_{q}=\mathbf{H}_{q}+\beta \operatorname{diag}\left(\mathbf{H}_{q}\right)$;

3. Update $\boldsymbol{q}: \boldsymbol{q}_{i+1}=\boldsymbol{q}_{i}-\hat{\mathbf{H}}_{q}^{-1} \nabla_{q} \chi_{\mathbf{1}}^{2}\left(\boldsymbol{q}_{i}, \boldsymbol{\alpha}_{i}\right)$;

4. Update $\boldsymbol{\alpha}: \boldsymbol{\alpha}_{i+1}=\operatorname{prox}_{p, \lambda, \mathbf{W}}\left[\boldsymbol{S}_{\mathrm{atm}}\left(\boldsymbol{q}_{i+1}\right)-\boldsymbol{O}\right]$ using Alg. 1 every $k$ iterations.

end

return $q, \alpha$

Algorithm 2: Proximal Levenberg-Marquardt algorithm for analysis penalty.

Data: Stokes profiles, model atmosphere, transform $\mathbf{W}$ and $k$.

Result: Regularized solution

Initialization: $\boldsymbol{q}_{0}$ and $\boldsymbol{\alpha}_{0}=0$, first estimation of solution;

while not converged do

1. Compute gradient $\nabla_{q} \chi_{\mathbf{W}^{\mathrm{T}}}^{2}\left(\boldsymbol{q}_{i}, \boldsymbol{\alpha}_{i}\right)$ and Hessian

matrices $\mathbf{H}_{q}$;

2. Modify Hessians: $\hat{\mathbf{H}}_{q}=\mathbf{H}_{q}+\beta \operatorname{diag}\left(\mathbf{H}_{q}\right)$;

3. Update $\boldsymbol{q}: \boldsymbol{q}_{i+1}=\boldsymbol{q}_{i}-\hat{\mathbf{H}}_{q}^{-1} \nabla_{q} \chi_{\mathbf{W}^{\mathrm{T}}}^{2}\left(\boldsymbol{q}_{i}, \boldsymbol{\alpha}_{i}\right)$;

end

4. Update $\boldsymbol{\alpha}: \boldsymbol{\alpha}_{i+1}=\operatorname{prox}_{p, \lambda, \tau}\left[\boldsymbol{\alpha}_{i}-\tau \nabla_{\alpha} \chi_{\mathbf{W}^{\mathrm{T}}}^{2}\left(\boldsymbol{q}_{i+1}, \boldsymbol{\alpha}_{i}\right)\right]$;

return $q, \alpha$

Algorithm 3: Proximal Levenberg-Marquardt algorithm for synthesis penalty.

of clarity, we only focus on Stokes $I$, for which the systematic effects are usually more important. Throughout this section, we use the $\ell_{0}$-norm as regularization, so we apply the proximal operator of Eq. (13). The codes used in this paper can be obtained online ${ }^{5}$.

\subsection{Observations}

To show examples of application, we choose two spectral regions of special interest that suffer from the problems described in this paper. The first one is the region around $10830 \AA$, which contains the He I multiplet at $10830 \AA$ and is displayed in the right panel of Fig. 1. This multiplet is used for diagnosing the magnetic properties of chromospheric material. Owing to the potentially large Doppler shifts in the chromosphere (e.g., Lagg et al. 2007), it is interesting to apply our approach to this multiplet.

The observations that we analyze were obtained with the Vacuum Tower Telescope (VTT) on the Observatorio del Teide with the TIP-II instrument (Collados et al. 2007). The profiles were extracted from a plage region close to a pore. The He I multiplet is synthesized with the help of HAZEL (Asensio Ramos et al. 2008), which gives the $\boldsymbol{S}_{\mathrm{atm}}(\boldsymbol{q})$ part of Eq. (1). The spectral lines are characterized by the optical depth on the red component of the multiplet $\Delta \tau$, the Doppler broadening of the line $v_{\text {th }}$, and the bulk velocity of the plasma $v$.

The second region is the well-known region around 6301-6302 $\AA$ that contains an Fe I doublet. This region also contains two telluric absorptions. The Stokes profiles, shown in

\footnotetext{
5 https://github.com/aasensio/proxStokesSystematics
} 
the left panel of Fig. 1, have been obtained from the ground with the POlarimetric LIttrow Spectrograph (POLIS; Beck et al. 2005) and belong to the observations of the quiet Sun analyzed by Martínez González et al. (2008).

To fit the profiles, we choose an atmosphere in LTE and we infer the depth stratification of the temperature, velocity along the line-of-sight, and microturbulent velocity. For this experiment, we fix the number of nodes of the parameterization: five nodes for the temperature, three for the bulk velocity and one for the microturbulent velocity. Like virtually all inversion codes with depth stratification (e.g., Ruiz Cobo \& del Toro Iniesta 1992), we place the nodes equispaced in the $\log \tau_{\mathrm{c}}$ axis, with $\tau_{\mathrm{c}}$ the continuum optical depth at $5000 \AA$.

\subsection{Orthogonal wavelet regularization}

The orthogonal wavelet transform (Jensen \& la Cour-Harbo 2001) is a very powerful sparsity-inducing transformation in cases in which the signal is smooth. One of the advantages of the orthogonal wavelet transform is that a fast algorithm to compute the direct and inverse transformation exists, without ever computing the transformation matrix $\mathbf{W}$.

The specific approach for orthogonal transformations is irrelevant because both the analysis and synthesis penalties are equivalent. For convenience, we choose to do this study under the analysis penalty case. We impose the sparsity constraint using the transformation matrix $\mathbf{W}$ associated with the Daubechies- 8 orthogonal wavelet and for different values of the regularization parameter $\lambda$. The results are shown in Fig. 2. The left panel displays the observed data, the final fit (including systematic effects) and the inferred systematic effects. The right panel shows the wavelet coefficients of the systematic effects, showing only the first 100 coefficients of the potential 512 coefficients (the wavelength axis contains 512 sampled points). Each panel contains the percentage of active (non-zero) wavelet coefficients. We note that the fit quality is strongly affected by the value of the regularization parameters $\lambda$. The important point is that it is possible to find values of $\lambda$ that lead to a good fit of the He I multiplet and simultaneously fit the systematic effects. Of special relevance, in this case, is the extended wing of the Si I, which sometimes makes it difficult to set a continuum level for the $10830 \AA$ multiplet. Using this approach, the continuum level is automatically obtained from the fit.

When $\lambda$ is small, a significant overfitting of the data occurs, in which even the noise is absorbed by the systematic effects. For very large $\lambda$, the method cannot fit the observations. For intermediate values of $\lambda$, a very nice fit is obtained. The optimal thresholding of the wavelet coefficients is similar to the expected noise level, as pointed out by Starck et al. (2010). Using only $10 \%$ of the wavelet coefficients is probably enough to have a fit of the whole spectral region. It is interesting that the model that we impose for the He I multiplet is probably not enough to explain the observations at the noise level. This is the reason why some "extra absorption" is added by the systematic effects to the wings of the red component. If this behavior is undesirable, it is potentially possible to introduce an extra regularization to avoid them (for instance, an additional $\ell_{0}$ or $\ell_{1}$ regularization for the vector $\boldsymbol{\alpha}$ ). However, we demonstrate in the following that a better option is to regularize using different $\mathbf{W}$ transformations.

\subsection{Isotropic undecimated wavelet regularization}

The isotropic undecimated wavelet transform (IUWT; Starck \& Murtagh 1994; Starck et al. 2010) algorithm is a non-orthogonal redundant multiscale transform that is well suited to objects that are more or less isotropic. This transform has found great success for the denoising of astronomical images (e.g., Starck et al. 2010, and references therein). Recently, we have witnessed examples of applications to one-dimensional spectra (Machado et al. 2013). Given that this is a redundant non-orthogonal transform, the analysis penalty approach is more efficient from a computational point of view.

The IUWT can be efficiently applied using the à-trous algorithm, which proceeds as follows: The original data $I(\lambda)$ are filtered, in our case using the $\mathrm{B}_{3}$-spline given by the filter $[1,4,6,4,1] / 16$. The filtered data are substracted from the original ones, obtaining what is commonly known as the detail, $w_{i}(\lambda)$. The filtered data are again iteratively filtered (with scaled filters) up to a depth $d$, computing the detail in each scale. The final smoothed signal will be termed $c_{d}(\lambda)$. At the end, we have a smoothed version of the original signal and a set of details at each depth. The size of the IUWT is $d+1$ times the original data, so the information is really encoded on the correlation among the transformed coefficients. The data are reconstructed from the transformed data simply using

$I(\lambda)=c_{d}(\lambda)+\sum_{i=1}^{d} w_{i}(\lambda)$.

The results shown in Fig. 3 for the He I multiplet and Fig. 4 for the Fe I doublet have been obtained using the IUWT up to $d=6$ and applying the hard thresholding operator to all the details. The left panel shows the results when the full Alg. 1 is used to apply the proximal algorithm, while the right panels give the result when only the first iteration of this algorithm is used, which corresponds to using Eq. (15). The application of Eq. (15) cannot assure convergence to the correct solution to the problem, but we have tested that it does a very good job. This simplifies the application of our proposed algorithm to any existing inversion code because Eq. (15) can be implemented in only one or a few lines of code.

When the value of $\lambda$ is small, we witness the overfitting again, which also includes the addition of some broadening of the red component of the He I. When $\lambda$ is large, the fit is of low quality. In the intermediate range close to the expected noise standard deviation, we find an excellent fit of the whole profile, with a flat contribution exactly where the He I multiplet is located.

It is interesting to note that we have empirically found that applying Eq. (15) instead of solving the full problem via Alg. 1 gives better and more robust results (see Figs. 3 and 4). The reason has to be due to the fact that we are solving the optimization problem of Eqs. (5) or (7) by separating it into two simpler problems via an alternating optimization method. It is a general characteristic of these methods that they work better in practice if none of the two problems is solved with full precision at each iteration, but only approximately. If any of the two problems is solved with precision in any iteration, one can produce some amount of overfitting that is very difficult to compensate for in later iterations.

\subsection{Voigt functions}

The final example uses another non-orthogonal and redundant transformation, in this case not of general applicability, but 
A. Asensio Ramos et al.: Inversion of Stokes profiles with systematic effects
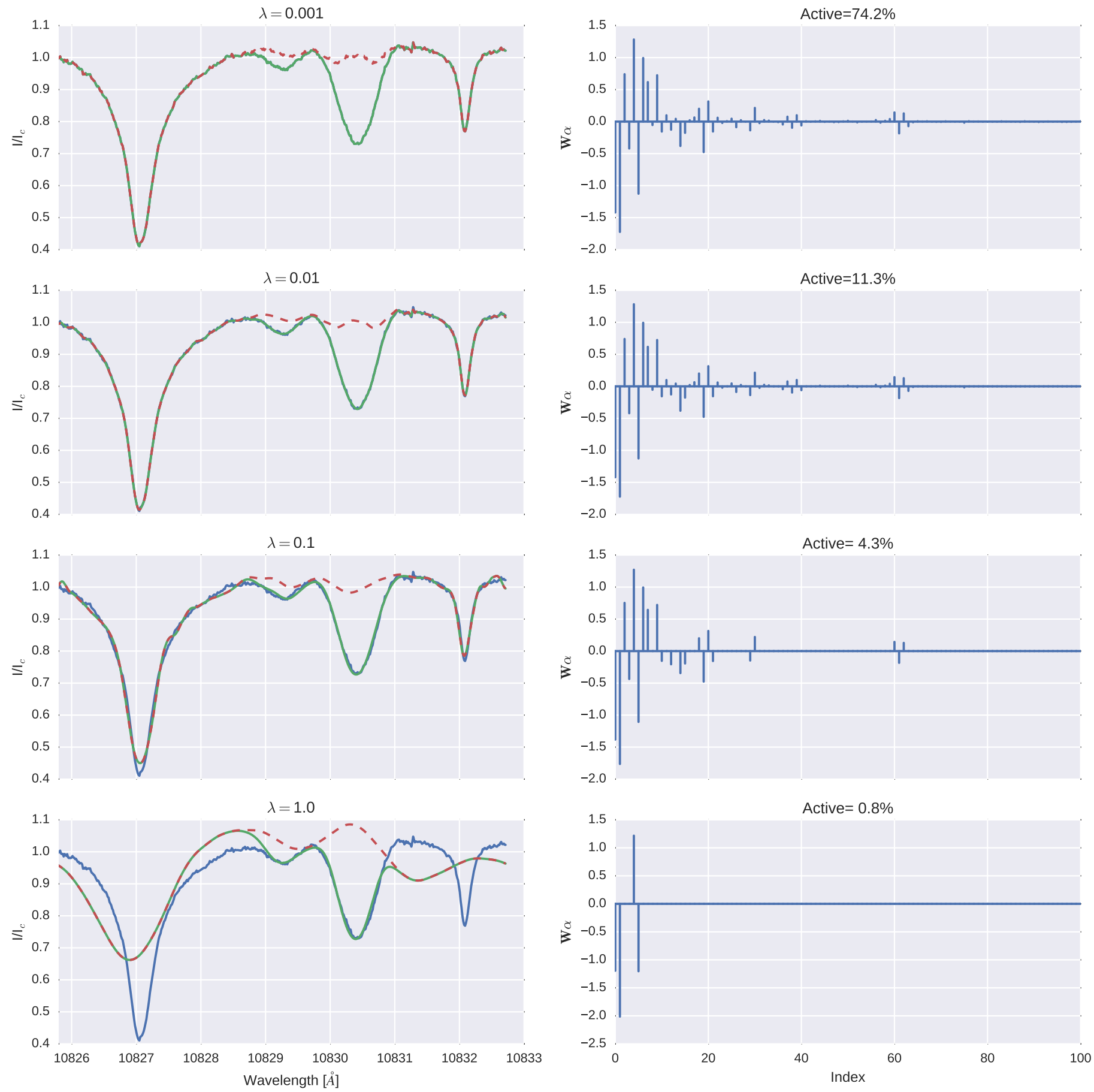

Fig. 2. Left panels: observed (blue) and fitted (green) Stokes $I$ profile for different values of the regularization parameters $\lambda$ and using an orthogonal wavelet transform as the sparsity-inducing transformation. The dashed red curve shows the inferred systematic effects. The spectral range corresponds to that around the He I multiplet at $10830 \AA$. Right panels: first 100 active wavelet coefficients of the set of 512 total coefficients. The percentage of active functions is shown in each panel.

tailored to explain the systematic effects. The Hermite functions described by del Toro Iniesta \& López Ariste (2003) is a good option, but we prefer to utilize a basis set made of Voigt functions centered at every sampled wavelength point:

$\phi_{i}(\lambda)=H\left(\frac{\lambda-\lambda_{i}}{\Delta}, a\right)$

Even though the basis is non-orthogonal, it is much easier in this case to work in the synthesis prior approach, because the results are much more transparent. The reason is that we directly impose the sparsity constraint on the coefficients associated with all the Voigt functions.

In this case, we focus on the 6301-6302 A spectral region. The systematic effects are modeled with Voigt functions placed at every sampled wavelength between 6301.15 and $6302.93 \AA$, which includes both $\mathrm{Fe}$ I lines and both telluric contaminations. The damping constant is fixed to $a=0.8$, which gives line wings similar to those observed in the telluric lines. To boost sparsity, 

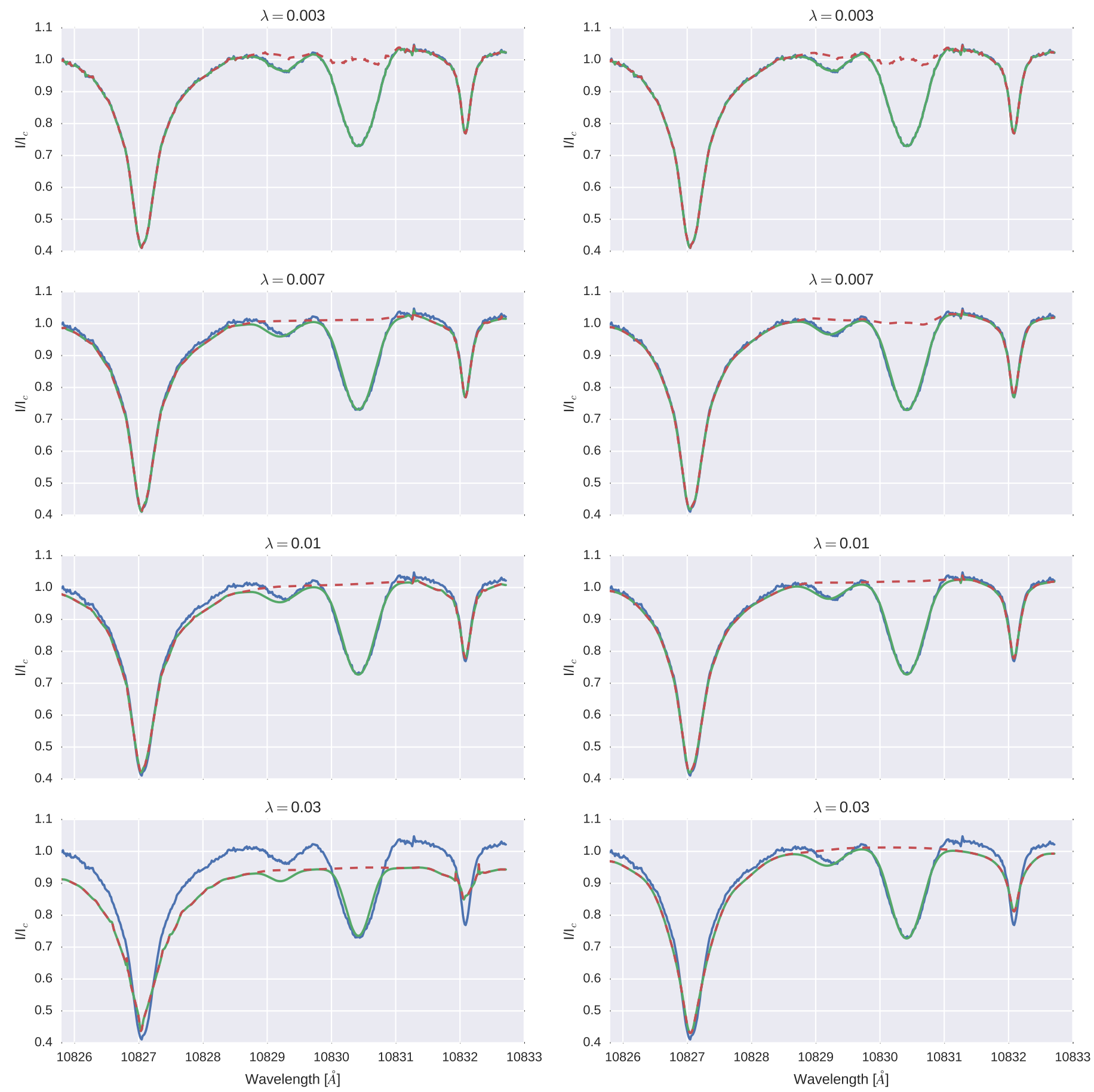

Fig. 3. Observed (blue), fitted (green) and systematic effects (dashed red) obtained using the IUWT in the region around $10830 \AA$ and using the updated version of HAZEL. The left panels correspond to using the full Alg. 1, while the right panel shows the results when using the simplified Eq. (15).

we consider two different widths, $\Delta=14.8,29.6 \mathrm{~m} \AA$, although more fits and damping parameters can be easily considered with ease.

The results are displayed in Fig. 5 for different values of $\lambda$. The left panel shows the observations and the fit, together with the inferred systematic effects. The right panel shows the active Voigt functions. When $\lambda$ is too small, a very good fitting is found, but we can see that the optimization induces corrections on the spectral lines of interest. This is, in principle, undesired, although it is clearly stating that the model proposed for the spectral lines cannot fit them with enough precision. When $\lambda$ is large, the fit of the systematic effects is bad, which negatively impacts the fit of the spectral lines of interest. For intermediate values of the regularization parameter, we find an acceptable fit, where only the telluric absorption lines are fitted by the systematic effects with a very sparse solution.

\section{Conclusions}

In this study, we have developed a new method that enables systematic effects to be dealt with in data inversions. These systematic effects can include a variety of calibration defects, spectral 

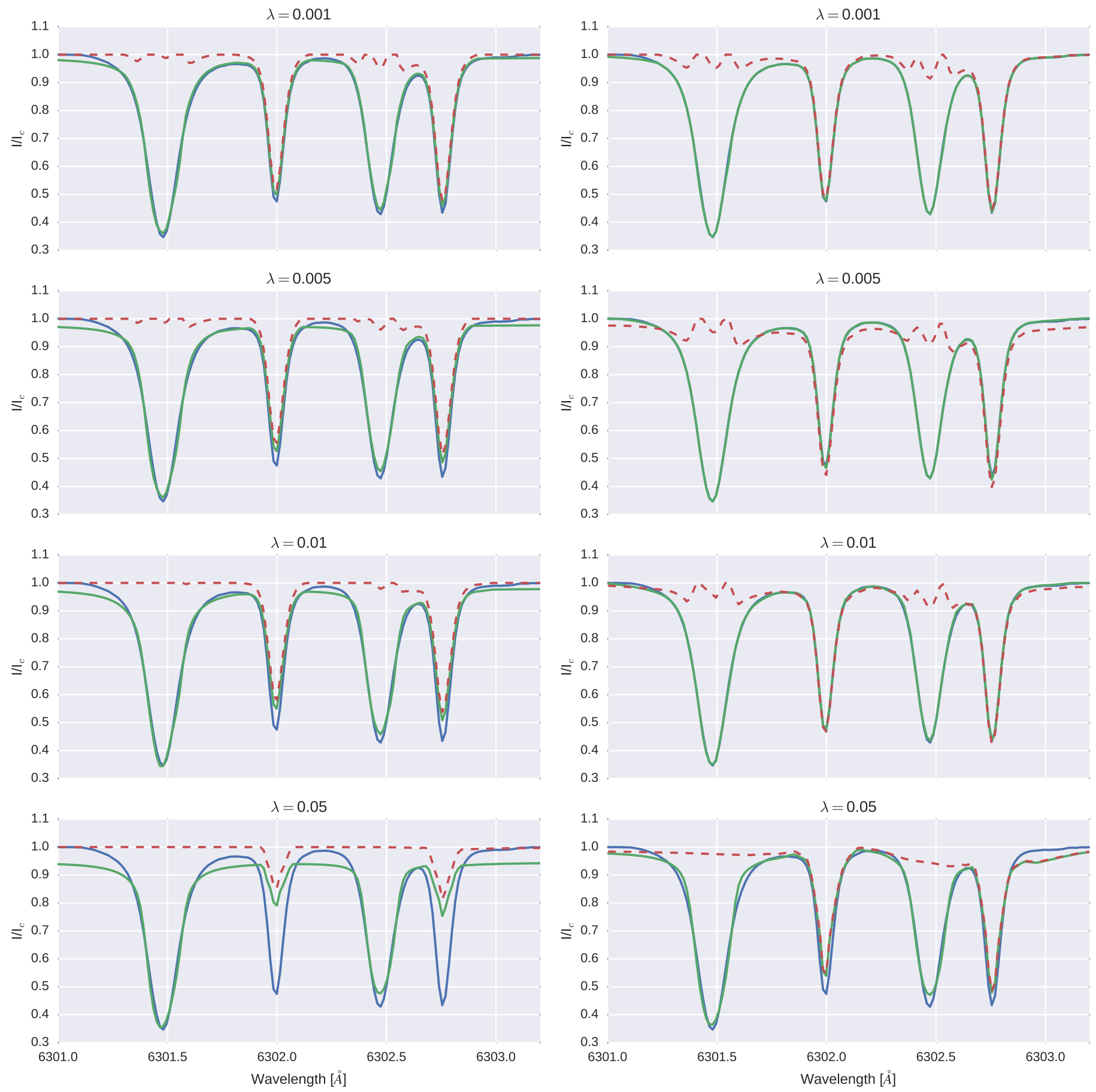

Fig. 4. Observed (blue), fitted (green), and systematic effects (dashed red) obtained using the IUWT in the 6301-6302 A spectral region and using the inversion code that assumes local thermodynamic equilibrium. The left panels correspond to using the full Alg. 1, while the right panel shows the results when using the simplified Eq. (15).

lines from the Earth's atmosphere, or spectral features that are present in the observations that our model atmosphere cannot reproduce. Our method builds upon the assumption that these systematic effects are not random noise and, therefore, they have an inherent level of sparsity in a suitable basis set.

We have shown that the assumption of sparsity induces a very powerful regularization because the resulting cross-talk between the inversion model and the systematic effects can be made very low. To do so, we have introduced a regularization parameter $\lambda$ that must be adjusted according to the needs of each problem based on experience. However, we argue that this step is not very different from selecting the weights of the observations in current implementations of inversion codes and a rough estimate can be obtained a priori.

Although the mathematical characterization of the algorithm can appear a bit convoluted and depends on recent advances on the optimization of non-smooth functions, a few extra lines of code should suffice to make it work with current inversion codes. Our experience with the SIR and HAZEL codes has positively confirmed this. When these systematic effects are not corrected, inversion codes usually try to overcompensate their effect by converging the parameters of the model to a wrong solution. Our 

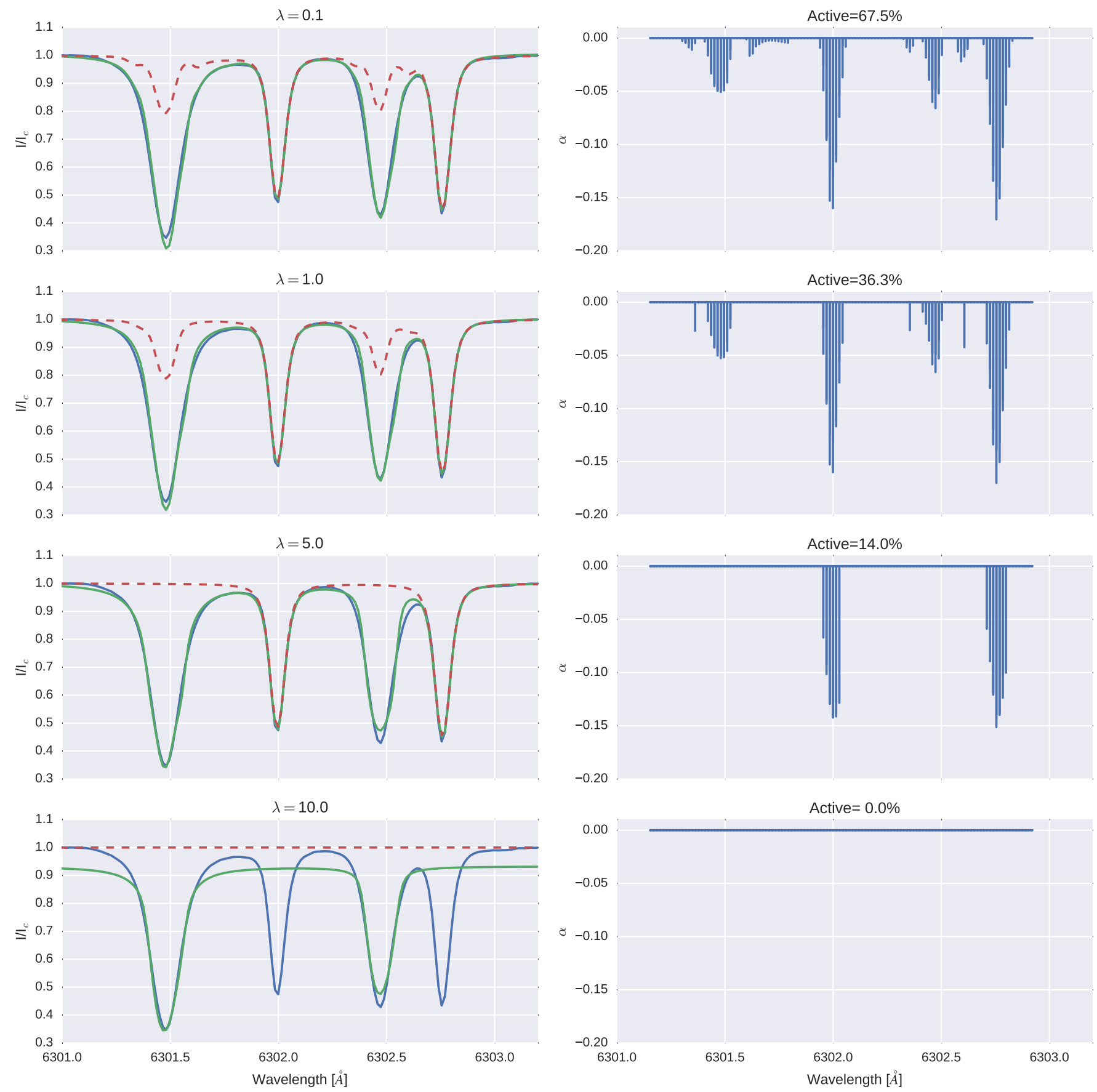

Fig. 5. Observed (blue) and fitted (green) Stokes $I$ profile for different values of the regularization parameters $\lambda$ and using a dictionary made of Voigt functions centered at every pixel. The dashed red curve shows the inferred systematic effects. The left panels show the results for the $\mathrm{Fe} \mathrm{I}$ lines, while the right panels show the active functions, with the label indicating the percentage of active functions.

method minimizes the impact of such effects in the convergence of the solution when an adequate value of the regularization parameter is selected.

Our method has a few interesting advantages over the downweighting technique for dealing with systematic effects. Arguably the most important one concerns the assumptions imposed on the generative model. Using the $\chi^{2}$ merit function requires that the uncertainty of the residual between the observations and the model is Gaussian with zero mean and a certain variance. This is not true unless one is able to model all expected signals. Only if everything is modeled (to a certain level, of course), can one be sure that the results can be interpreted appropriately (for instance, error bars). A second advantage concerns the reduction in the subjectivity on the election of parameters. One only needs to choose the value of $\lambda$ and the method automatically adapts the solution to the systematic effects.

Acknowledgements. Financial support by the Spanish Ministry of Economy and Competitiveness through projects AYA2014-60476-P, AYA2014-60833$\mathrm{P}$, and Consolider-Ingenio 2010 CSD2009-00038 are gratefully acknowledged. J.d.1.C.R. is supported by grants from the Swedish Research Council (Vetenskapsrådet) and the Swedish National Space Board. A.A.R. also acknowledges financial support through the Ramón y Cajal fellowship. This research has made use of NASA's Astrophysics Data System Bibliographic Services. This 
article is based on observations taken with the VTT telescope operated on the island of Tenerife by the Kiepenheuer-Institut für Sonnenphysik in the Spanish Observatorio del Teide of the Instituto de Astrofísica de Canarias (IAC). We acknowledge the community effort devoted to the development of the following open-source Python packages that were used in this work: numpy, matplotlib, scipy, seaborn and PyWavelets.

\section{References}

Asensio Ramos, A. 2009, ApJ, 701, 1032

Asensio Ramos, A., \& de la Cruz Rodríguez, J. 2015, A\&A, 577, A140

Asensio Ramos, A., Martínez González, M. J., \& Rubiño Martín, J. A. 2007, A\&A, 476, 959

Asensio Ramos, A., Trujillo Bueno, J., \& Landi Degl'Innocenti, E. 2008, ApJ, 683,542

Asensio Ramos, A., Manso Sainz, R., Martínez González, M. J., et al. 2012, ApJ 748,83

Auer, L. H., House, L. L., \& Heasley, J. N. 1977, Sol. Phys., 55, 47

Beck, A., \& Teboulle, M. 2009, SIAM J. Img. Sci., 2, 183

Beck, C., Schmidt, W., Kentischer, T., \& Elmore, D. 2005, A\&A, 437, 1159

Borrero, J. M., Tomczyk, S., Norton, A., et al. 2007, Sol. Phys., 240, 177

Borrero, J. M., Tomczyk, S., Kubo, M., et al. 2010, Sol. Phys., 35

Candès, E., Romberg, J., \& Tao, T. 2006, Comm. Pure Appl. Math., 59, 1207

Collados, M., Lagg, A., Díaz García, J. J., et al. 2007, in The Physics of Chromospheric Plasmas, eds. P. Heinzel, I. Dorotovič, \& R. J. Rutten, ASP Conf. Ser., 368, 611

del Toro Iniesta, J. C., \& López Ariste, A. 2003, A\&A, 412, 875

Donoho, D. 2006, IEEE Trans. on Information Theory, 52, 1289

Elad, M., Milanfar, P., \& Rubinstein, R. 2007, Inverse Problems, 23, 947

Fadili, M. J., \& Starck, J.-L. 2009, in Proc. IEEE International Conference on Image Processing

Harvey, J., Livingston, W., \& Slaughter, C. 1972, in Line Formation in the Presence of Magnetic Fields, 227

Jensen, A., \& la Cour-Harbo, A. 2001, Ripples in Mathematics: The Discrete Wavelet Transform (Springer Verlag)

Lagg, A., Woch, J., Solanki, S. K., \& Krupp, N. 2007, A\&A, 462, 1147

Landi Degl'Innocenti, E., \& Landi Degl'Innocenti, M. 1977, A\&A, 56, 111

Landi Degl'Innocenti, E., \& Landolfi, M. 2004, Polarization in Spectral Lines (Kluwer Academic Publishers)

Lee, J. D., Sun, Y., \& Saunders, M. A. 2012, SIAM J. Opt., 24, 3

Levenberg, K. 1944, Q. J. Math, 2, 164

Lites, B., Casini, R., Garcia, J., \& Socas-Navarro, H. 2007, Mem. Soc. Astron. It., 78,148

Machado, D. P., Leonard, A., Starck, J.-L., Abdalla, F. B., \& Jouvel, S. 2013, A\&A, 560, A83

Marquardt, D. 1963, SIAM J. Appl. Math., 11, 431

Martínez González, M. J., Collados, M., Ruiz Cobo, B., \& Beck, C. 2008, A\&A, 477, 953

Orozco Suárez, D., Bellot Rubio, L. R., del Toro Iniesta, J. C., et al. 2007, ApJ, 670,61

Parikh, N., \& Boyd, S. 2014, Foundations and Trends in Optimization, 1
Ruiz Cobo, B., \& Asensio Ramos, A. 2013, A\&A, 549, L4 Ruiz Cobo, B., \& del Toro Iniesta, J. C. 1992, ApJ, 398, 375 Ruiz Cobo, B., \& del Toro Iniesta, J. C. 1994, A\&A, 283, 129 Sanchez Almeida, J. 1992, Sol. Phys., 137, 1

Skumanich, A., \& Lites, B. W. 1987, ApJ, 322, 473

Socas-Navarro, H., Trujillo Bueno, J., \& Ruiz Cobo, B. 2000, ApJ, 530, 977 Socas-Navarro, H., de la Cruz Rodriguez, J., Asensio Ramos, A., Trujillo Bueno, J., \& Ruiz Cobo, B. 2015, A\&A, 577, A7

Starck, J.-L., \& Murtagh, F. 1994, A\&A, 288, 342

Starck, J.-L., Murtagh, F., \& Fadili, J. M. 2010, Sparse image and signal processing (Cambridge: Cambridge University Press)

van Noort, M. 2012, A\&A, 548, A5

\section{Appendix A: Second-order iterative scheme in the synthesis prior}

It is possible to use a second-order iterative scheme that would improve over Eq. (12) when using the synthesis prior approach. Instead of a simple step $\tau$, one uses the full Hessian, so that the iterative scheme is updated to read (Lee et al. 2012)

$\boldsymbol{\alpha}_{i+1}=\operatorname{prox}_{p, \lambda}^{H_{\alpha}}\left[\boldsymbol{\alpha}_{i}-\mathbf{H}_{\alpha}^{-1} \nabla_{\alpha} \chi_{\mathbf{W}^{\mathrm{T}}}^{2}\left(\boldsymbol{q}_{i+1}, \boldsymbol{\alpha}_{i}\right)\right]$ with $\boldsymbol{q}_{i+1}$ fixed.

The main obstacle is the computation of the so-called scaled proximal operator $\operatorname{prox}_{p, \lambda}^{H_{\alpha}}(\boldsymbol{\alpha})$. It can be demonstrated that this proximal operator can be efficiently computed using the accelerated FISTA iteration (Beck \& Teboulle 2009), which is displayed in Alg. 4.

Data: $\mathbf{H}_{\alpha}$ and $\boldsymbol{\alpha}$

Result: $\operatorname{prox}_{h}^{H}(\boldsymbol{\alpha})$

Initialization: $t_{0}=1, \boldsymbol{y}_{1}=\alpha$ and step $\tau<2 /\left\|\mathbf{H}_{\alpha}\right\|^{2}$;

while not converged do

1. Update values: $\boldsymbol{y}_{i+1}^{\prime}=\boldsymbol{y}_{i}-\tau \mathbf{H}_{\alpha}\left(\boldsymbol{y}_{i}-\boldsymbol{\alpha}\right)$;

2. Apply projection: $\boldsymbol{\alpha}_{i+1}=\operatorname{prox}_{p, \lambda, \tau}\left(\boldsymbol{y}_{i+1}^{\prime}\right)$;

3. Update weight: $t_{i+1}=\frac{1+\sqrt{1+4 t_{i}^{2}}}{2}$;

4. Update solution: $\boldsymbol{y}_{i+1}=\boldsymbol{y}_{i}+\left(\frac{t_{i}-1}{t_{i+1}}\right)\left(\boldsymbol{y}_{i+1}^{\prime}-\boldsymbol{y}_{i}\right)$;

end

return $y_{i}$

Algorithm 4: FISTA algorithm for scaled proximal projection. 